\title{
Le tambour rẽ et son pouvoir
}

\section{Michael Oppitz}

Traducteur : Isabelle Schulte-Tenckhoff

\section{OpenEdition}

\section{Journals}

Édition électronique

URL : http://journals.openedition.org/ethnomusicologie/2382

ISSN : 2235-7688

\section{Éditeur}

ADEM - Ateliers d'ethnomusicologie

\section{Édition imprimée}

Date de publication : 1 janvier 1990

Pagination : 79-95

ISBN : 2-8257-0423-7

ISSN : 1662-372X

\section{Référence électronique}

Michael Oppitz, « Le tambour rẽ et son pouvoir », Cahiers d'ethnomusicologie [En ligne], 3 | 1990, mis en ligne le 15 octobre 2011, consulté le 19 avril 2019. URL : http://journals.openedition.org/ ethnomusicologie/2382 


\title{
LE TAMBOUR RẼ ET SON POUVOIR*
}

\author{
Michael Oppitz
}

Le tambour $r e \tilde{e}$ est réparti dans à une aire relativement restreinte de l'Himalaya népalais, ayant pour centre le pays des Magar septentrionaux établis au sudouest du massif de Dhaulagiri. Les membres de ce groupe ethnique par ailleurs hétérogène connaissent la même langue, le kham (c'est pourquoi on les appelle aussi Kham Magar), le même système d'alliance avec mariage préférentiel de cousins croisés matrilatéraux, ainsi que des pratiques religieuses communes que l'on peut qualifier de chamaniques au sens strict, c'est-à-dire proches de celles du domaine sibérien. Les chamanes des Magar septentrionaux possèdent un attirail unique dont l'élément essentiel est le tambour magique rẽ qui apparaît comme un véritable emblème de la profession.

Après une brève description morphologique de l'instrument, je retracerai le destin d'un tambour $r e \tilde{a}$ à travers le temps, sa biographie pour ainsi dire, pour examiner enfin ses nombreuses fonctions. Car c'est par elles que s'expriment les pouvoirs inhérents au tambour en tant que moyen de communication avec l'Au-delà. En effet, aux yeux de ceux qui le fabriquent et en jouent, le tambour $r e \tilde{e}$ représente plus qu'un simple instrument de musique, car il permet de fréquenter les forces surnaturelles, de les mettre à contribution ou de les repousser, selon les cas - bref, de les faire agir en faveur du commun des mortels d'une manière inaccessible à celui-ci. C'est seulement en tenant compte de cette dimension magique et religieuse du tambour chamanique qu'il devient possible de lui donner toute sa valeur muséographique ${ }^{1}$.

Traduit de l'allemand par Isabelle Schulte-Tenckhoff.

Je n'aurais jamais eu l'idée d'écrire le texte qui suit sans l'encouragement de Mireille Helffer qui a deviné, il y a longtemps, le caractère unique du tambour rẽ. 


\section{Le tambour rẽ et ses éléments}

En langue kham, le terme rẽ désigne le tambour sur cadre à membrane unique du chamane. On rencontre aussi le terme binômial de rẽgor qui ne désigne au sens strict que le «cadre du tambour ${ }^{2}$ mais qui peut se référer aussi au tambour tout entier ${ }^{3}$. Dans la langue des Chepang du Népal central, on retrouve le mot $r \tilde{e}$ à la place de ring, nom qui s'applique également au tambour sur cadre à membrane unique. Le tambour rê est formé de divers éléments nommés et fabriqués avec des matériaux prescrits par la mythologie:

- un cadre (rẽgor) fait d'une latte découpée dans le bois d'un chêne épineux (gui ou guipāl), d'où la dénomination guipal rẽgor;

- quelques clous (killi) et plaquettes ( tãs) en fer, appliqués en rangs verticaux, pour river les deux extrémités effilées de la latte de bois;

- une membrane (syelo) en peau de chèvre sauvage de l'Himalaya (Nemorhaedus goral; sahr en kham);

- diverses lanières en cuir ( $\bar{t} a n \bar{a})$ pour lacer la membrane;

- un certain nombre de trous percés au feu (dulo) sur le pourtour ouvert du cadre, servant à tendre les lanières retenant la membrane;

- un cerceau en tendon élastique ( $y e l$ ) entourant le cadre du côté où est fixée la membrane, pour éviter que les lanières ne se déplacent;

- deux baguettes de bambou en forme de X (hâtāsso), suspendues à des chaînettes en fer attachées à l'intérieur du cadre, servant à tenir l'instrument à la main;

- quatre chaînettes en fer (sāngal) à deux ou trois maillons servant à lier les poignées en bambou au cadre;

- une baguette ( $g \bar{a} j a$ ) en bois māke; en raison du matériau employé, celle-ci est aussi désignée dans la mythologie par le terme «cuiller en bois de māke» (māke dabli).

A cette liste, il faut ajouter le panier allongé $(p \bar{e})$ en bambou tressé, muni d'un couvercle, dans lequel repose l'instrument à côté d'autres accessoires de l'attirail chamanique, ainsi que le sac en chanvre à bandoulière (zã̃) dans lequel l'assistant du chamane transporte le panier sur le lieu de la séance, après l'avoir enveloppé d'une couverture de laine. Quand il n'est pas joué, le tambour des Magar est ainsi soustrait aux regards, contrairement aux tambours correspon-

2 Le suffixe -gor, apparenté au tibérain khor (= cercle, périmètre, cerceau), se réfère à un cercle ou à un cadre rond.

3 Les Magar connaissent un troisième terme pour le tambour chamanique, celui de dhyāngro, répandu dans tout le Népal avec les variantes dhãgori, dhãgoro, dāngora et dãkura. En nepali, l'onomatopée dhyāng désigne un «son explosif», et il suggère la possibilité d'un rapport étymologique avec l'appellation du tambour. 
dants d'autres régions de l'Himalaya, que l'on transporte ouvertement et que leur propriétaire garde suspendus dans sa maison lorsqu'ils ne sont pas en usage.

A chaque élément constitutif du tambour correspond un chant spécifique relatant la quête primordiale du matériau approprié et la bonne manière de le travailler, si bien que tous les éléments possèdent leur propre mythe d'origine. L'ensemble de ces chants est désigné par le terme «chants de la fixation de la peau sur le tambour» (dhãgori murine kheti). C'est précisément lorsqu'un tambour neuf ou nécessitant réparation reçoit une nouvelle membrane qu'ils sont exécutés l'un après l'autre par un chanteur expérimenté et maître chamane. La longueur de ces chants variant entre trente et cent vers, ils sont nettement plus brefs que les grands mythes cosmogoniques. Mais ils visent moins à relater une histoire au scénario compliqué qu'à rappeler les étapes de la fabrication de l'instrument: ils s'apparentent ainsi à un mode d'emploi décrivant le prototype originel et donc le modèle de tous les tambours. Quant à leur structure métrique et à leur mode d'interprétation, ces chants sont cependant similaires aux mythes d'origine au contenu narratif plus riche.

La forme et les dimensions du tambour $r e \tilde{~ p e u v e n t ~ v a r i e r . ~ I l ~ e n ~ e x i s t e ~ t r o i s ~}$ types: circulaire, ovale et cardioïde. Répondant moins à un propos délibéré qu'aux contraintes résultant de la nature du bois et aux hasards du ploiement du cadre, ces formes semblent avoir la même valeur, bien que j'aie pu constater une certaine préférence esthétique pour la forme en cœur. Quant aux dimensions, elles varient entre 30 et $50 \mathrm{~cm}$ pour la hauteur, 30 et $45 \mathrm{~cm}$ pour la largeur, 105 et $150 \mathrm{~cm}$ pour la circonférence du cadre.

\section{Comparaisons}

Dans les villages des Magar septentrionaux, le tambour chamanique rẽ coexiste avec deux autres types de tambour, non chamaniques ceux-là, soit le dāmāye et le mãdal, dont le champ fonctionnel respectif est assez précis pour exclure tout recoupement. D'autre part, si les deux membranophones non chamaniques utilisés par les Magar sont de type pan-népalais, tel n'est pas le cas du tambour rẽ dont l'aire de diffusion est clairement délimitée. En dehors de celle-ci, un autre type de tambour chamanique est utilisé: le rnga ${ }^{4}$ des jhãkri (terme inter-régional désignant les guérisseurs).

4 Je réserve le terme de rnga à ce type de tambour, car sa facture montre une ressemblance frappante avec le tambour rnga du Tibet. Ce dernier, également un tambour sur cadre à deux membranes et muni d'un manche externe vertical, est largement répandu dans le Tibet bouddhiste et les pays voisins «lamaïsés »; il répond à de multiples usages dépassant les limites imposées par les différentes sectes. C'est le principal instrument à percussion des cérémonies des temples bouddhiques, des danses cham et des représentations de théâtre (Helffer 1983: 83); c'est aussi l'instrument indispensable des médiums spirituels tibétains (dpa'bo, lhapa) lors des séances extatiques (Berglie 1976: 94). Il existe bien évidemment quelques différences entre le tambour rnga du Tibet bouddhiste et le tambour des jhãkri du Népal central et oriental, mais il est hors de propos de les énumérer dans ces pages. 
Au-delà de quelques variations locales, ce second type possède les caractéristiques suivantes: c'est un tambour sur cadre circulaire à deux membranes en peau lacée et muni d'un manche faisant saillie verticalement et se terminant par une pyramide pointue ayant le plus souvent la forme d'un phurbu ou poignard magique tibétain. En règle générale, il est frappé avec une baguette en bambou recourbée en forme de $\mathrm{S}$.

Ainsi les deux types fondamentaux du tambour chamanique de l'Himalaya sont-ils diamètralement opposés du point de vue morphologique. Or, si le type oriental, c'est-à-dire le tambour des jhãkri, rappelle dans sa forme le tambour rnga du Tibet (fig. 1), on peut se demander quel serait le parent morphologique du type occidental, à savoir le tambour rẽ. Il n'est pas exagéré de le voir dans le tambour chamanique de l'Asie septentrionale - rapprochement qu'il convient toutefois de justifier pour éviter tout malentendu.

S'il est évident qu'un espace aussi vaste que l'aire du chamanisme «sibérien » classique (s'étendant du pays des Sami au détroit de Bering et de l'océan arctique au désert d'Asie centrale) a engendré quelque hétérogénéité, il n'en demeure pas moins vrai qu'il possède certains traits fondamentaux. En effet, le tambour chamanique de l'Eurasie septentrionale est muni d'une seule peau et, à quelques exceptions près ${ }^{5}$, de poignées fixées à l'intérieur du cadre (comme le rẽ des Magar) - poignées soit en matériau dur (bois, fer, corne) et attachées au cadre lui-même, soit en matériaux durs et flexibles à la fois (bois, fer et cuir, écorce, coton) et suspendues à l'intérieur du tambour. Ces deux types de poignées se répartissent grosso modo en deux aires, l'ouest pour les premières et l'est pour les secondes, leur zone de recoupement se situant entre $80^{\circ}$ et $90^{\circ}$ de longitude nord.

L'originalité de la facture du rẽ vient alors du fait que ce tambour combine les deux procédés: du point de vue du matériau, ses poignées tendent vers le type occidental; quant à leur fixation, en revanche, elles se rapprochent du type oriental. Le Népal se trouve dans la zone de recoupement, bien que nettement plus au sud - situation qui explique peut-être l'apparition d'un matériau (le bambou) absent des autres tambours nord-asiatiques. Du point de vue technique, la croix est également unique: ses bras ne forment pas l'angle droit caractéristique des manches fixes en fer ou en bois des tambours chamaniques «sibériens», mais un X. Ce qui confère au tambour rẽ un double mode de percussion: aux coups de baguette frappés sur la membrane s'ajoute l'entrechoc des croix en bambou suspendus à l'intérieur de l'instrument. Bien qu'il s'apparente au tambour sur cadre à membrane unique de type «sibérien », le tambour des Magar est donc une création ingénieuse de par la facture de ses poignées. Néanmoins, le tambour rẽ s'apparente davantage au tambour chamanique de l'Asie du Nord éloignée de milliers de kilomètres, qu'aux tambours des guérisseurs (jhãkri) du reste de l'Himalaya.

5 Pour les quelques cas de tambours au manche fixé à l'extérieur du cadre, voir Hoffmann (1950: 201-3), Lot-Falck (1961: 26-27), Haslund-Christensen (1944: 13-15) et Heissig (1944: 46 s. et pl. $1,4 \mathrm{a}, \mathrm{b}, 5)$. 


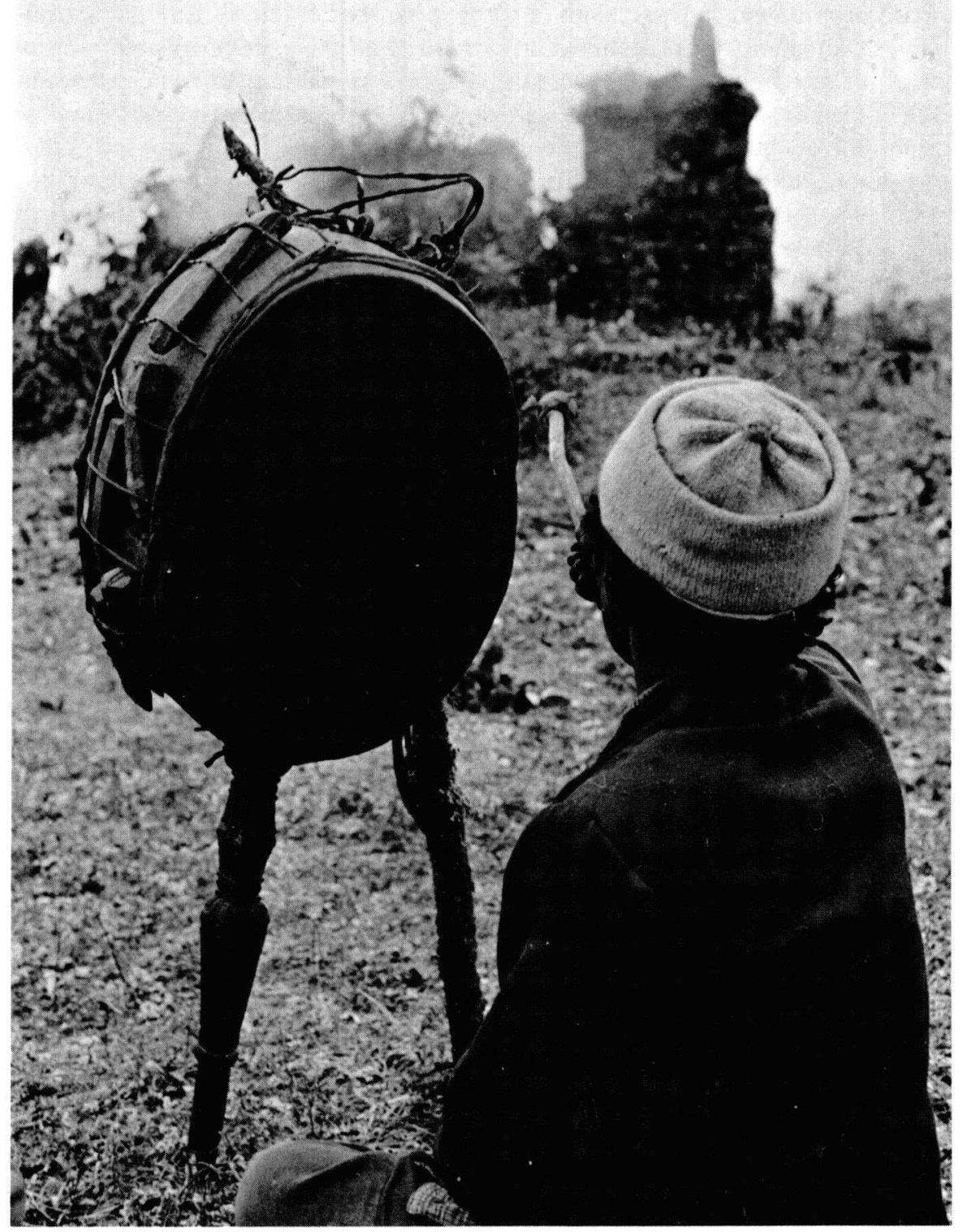

Fig. 1: Tambour rnga des Sherpa. Photo: M. Oppitz. 


\section{Biographie du tambour}

La fabrication d'un tambour rẽ se déroule en trois étapes différentes dans l'espace et le temps: tout d'abord, la recherche de l'arbre et la fabrication du cadre, puis le rivetage du cadre et la fixation des poignées, enfin l'application de la peau et la consécration du tambour fini. La première étape a lieu dans un espace proprement sauvage, dans la haute montagne et loin de tout habitat humain, la deuxième dans l'atelier d'un forgeron au village, et la troisième sur un carrefour peu fréquenté à la lisière du village, à mi-chemin entre l'espace sauvage et l'espace social. Ainsi, au cours de sa fabrication, le tambour oscille déjà entre l'univers humain et l'univers extra-humain.

Pour le novice, la période précédant l'initiation est marquée par des troubles psychiques et des rêves. C'est dans un rêve que lui apparaît l'arbre dans lequel il devra découper le cadre de son tambour. En règle générale, cet arbre se trouve dans une forêt en haute montagne, à plusieurs heures de marche du village. Un jour propice de pleine lune, un petit groupe constitué du novice, de deux chamanes et de sept assistants se rend dans la montagne pour découvrir l'arbre. Après avoir invoqué l'aide de l'esprit de son ancêtre, le novice s'étend à même le sol près d'une source et feint de dormir; une nouvelle vision lui fait reconnaître l'arbre qui lui est destiné. En état de transe, il avance ensuite à tâtons à travers le sous-bois. Lorsqu'il a identifié l'arbre (un chêne épineux), il exécute autour de lui des sauts de singe (possédé qu'il est par l'esprit auxiliaire du singe), puis se couche sous l'arbre en feignant à nouveau de dormir. Après avoir déposé des offrandes (levure, encens, banderoles en tissu, riz) au pied de l'arbre, les assistants se mettent à l'abattre à l'aide d'une hache. On veille à ce que l'arbre tombe en direction de l'est, qui est celle de la naissance et de la vie. Sur place, on ôte les branches. Puis les assistants enfourchent le tronc et l'emportent. Le verbe employé ici, soit chebne, «aller à cheval», s'apparente à la racine tibétaine chibs («cheval») et se réfère à la fonction ultérieure du tambour en tant que moyen de transport, «cheval», véhicule.

Au bord du chemin, le tronc est coupé en deux dans le sens de la longueur à l'aide d'un marteau en bois, d'une hache et de coins, et chaque moitié est taillée en forme de latte. A huit autres étapes, les deux lattes sont amincies davantage. En cours de route, le novice, qui ne participe à aucune activité manuelle, porte la hache en fer.

Au neuvième et dernier arrêt, sur un pâturage à 600 mètres au-dessus du village, un des assistants creuse dans le sol un trou rond dans lequel neuf bâtons en bois sont enfoncés pour former un second cercle intérieur plus petit. Entre-temps, un chamane a chauffé une des deux lattes au feu pour l'assouplir. Une fois qu'elle est assez flexible, les assistants l'insèrent dans l'espace entre le bord du trou et les neuf morceaux de bois. C'est le moment critique de l'opération. Si la latte se fend ou se casse, on la remplace par la seconde. Si tout va bien, on entoure provisoirement le point de chevauchement des deux extrémités du cadre recourbé sur lui-même avec des lanières de bambou, en attendant qu'il soit définitivement rivé. Une fois l'opération de courbure achevée, le cha- 
mane dirigeant l'expédition fait une offrande au dieu de la terre bhume, en y versant de l'eau de vie mada et du sang de poussin. Content du succès de l'opération, chaque assistant porte un toast au futur tambour: "Tous t'appelleront»; "On t'attend déjà avec des boucs gras»; «Tu rendras le novice célèbre et riche»; «Va pour lui dans le monde souterrain, Tambour».

Avant la tombée de la nuit, le groupe se dirige avec le nouveau cadre vers la lisière du village où l'attend déjà le maître chamane. Ici un autre trou a été creusé, dans lequel on fait «rouler» le cadre sur les derniers mètres du trajet. Ce geste est prescrit par la mythologie: il évoque les déplacements du futur tambour, ce véhicule des voyages dans le monde souterrain. Une fois le cadre logé dans le trou, le tout est couvert de terre pour la nuit. Cet enterrement du cadre représente le premier voyage souterrain du nouveau tambour, en prévision des tâches que, selon la coutume, il remplira dans les séances de guérison. Le lendemain matin, le cadre est déterré et, en présence du maître chamane, du novice et de quelques assistants, il est transporté chez le forgeron. A l'aide d'un clou chauffé au rouge, celui-ci perce tout d'abord quelques trous dans le cadre. Ils sont destinés aux lanières en cuir laçant la membrane, aux rivets de la jointure, et aux œillets en fer des poignées. Pour fermer le cadre, les deux extrémités sont maintenues ensemble avec une pince en fer (fig. 2). Trois plaquettes de fer disposées verticalement sont appliquées sur l'extrémité extérieure et deux sur l'extrémité intérieure. Une fois le tambour fini, ces rectangles bombés en forme de pyramide percés d'un œil au milieu représentent des points critiques de danger et de divination. Leur application selon les règles de l'art exige ainsi la plus haute attention du forgeron. Ensuite, celui-ci fixe à l'intérieur du cerceau les quatre œillets et les quatre chaînettes en fer destinés aux poignées. Enfin, il attache les deux croix en bambou aux chaînettes, après y avoir percé les trous nécessaires.

Ce jour-là, le forgeron agit en tant que Tiko Kami, forgeron mythique du monde souterrain. L'opération terminée, accompagné par les spectateurs et les assistants, il porte fièrement le cadre du tambour dans la maison du novice. Là, en échange de son travail, il reçoit de la nourriture, un billet de 5 roupies et un nouveau turban blanc. En quittant la forge, le maître chamane s'adresse ainsi à lui: «Frère aîné, Tiko Kami, forgeron du monde souterrain, ton travail sur le cadre du tambour a réussi ». Puis il interprète les vers suivants puisés dans le répertoire des chants mythiques:

«A l'aide des neuf assistants nous soulevons le tambour du monde souterrain.

Nous soulevons les âmes du monde souterrain.

Nous faisons l'ordre dans les impasses et dans les dangers.

Frère aîné, Tiko Kami, avec lẹ cadre sāndhan ${ }^{6}$ du tambour ».

$6 \quad$ Le terme de sāndhan s'apparente au népali sãdan qui signifie bois de santal, et l'expression sāndhan rẽgor coexiste avec guipal rẽgor pour désigner le cadre du tambour chamanique. Ce bois est prescrit dans l'Himalaya tibétain pour fabriquer des tambours (Helffer 1983: 68). Comme les Magar se servent exclusivement du bois du chêne épineux, l'emploi du terme de sāndhan revient au mieux à l'évocation, par le langage, d'un matériau prestigieux qui a peutêtre été employé jadis. 


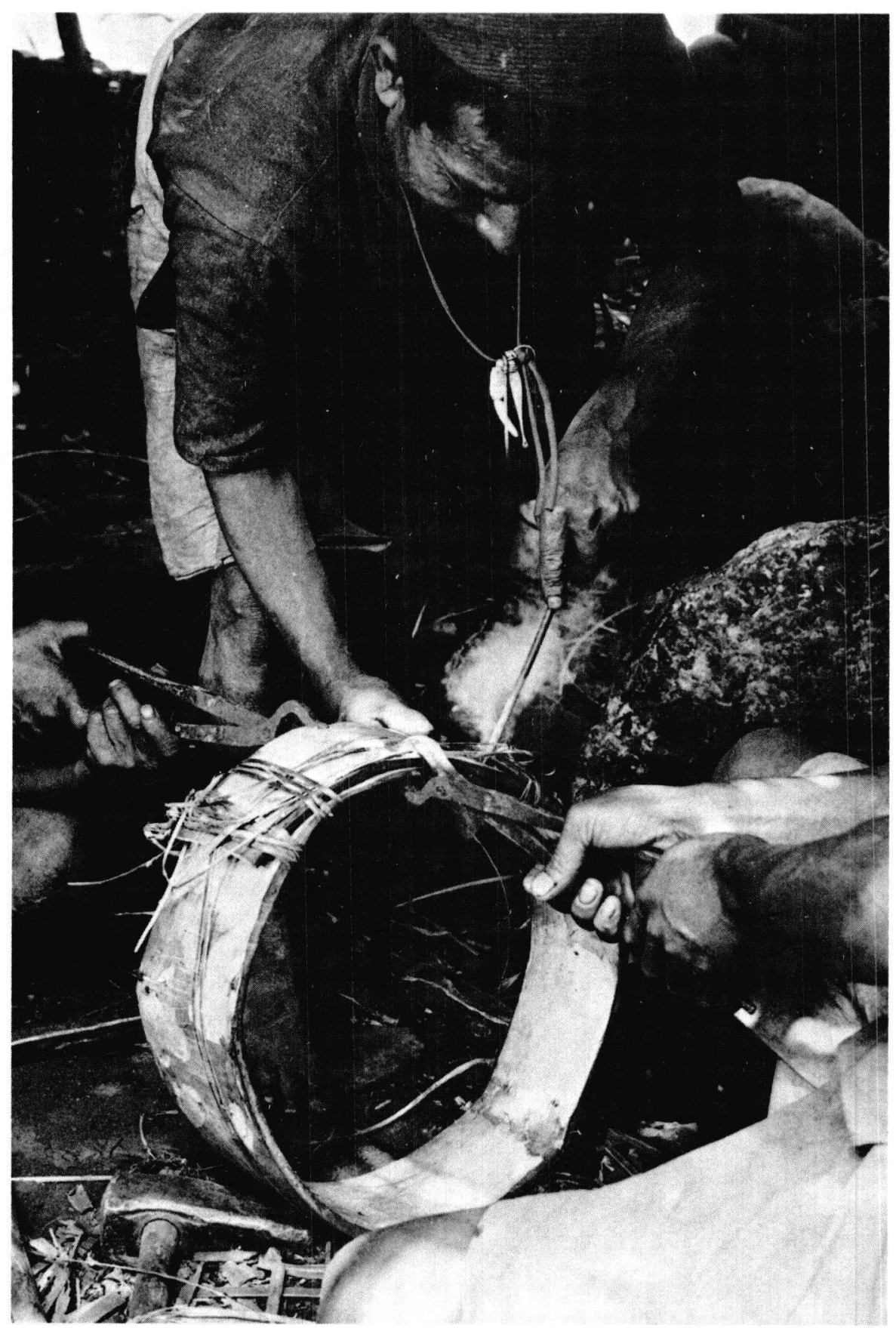

Fig. 2: Chez le forgeron: fixation des rivets en fer sur le chevauchement des deux extrémités du cadre du tambour. Photo: M. Oppitz. 
Un assistant bat le rythme avec un bâton sur le cadre du tambour encore dépourvu de membrane.

La fixation de la membrane est prévue un jour de pleine lune, comme la quête de l'arbre un mois plus tôt. Le propriétaire du tambour, quelques assistants et un chamane initié agissant comme maître de cérémonie se rencontrent sur un carrefour peu fréquenté près du village. S'il s'agit d'une réparation et non d'une première application, on ôte tout d'abord la membrane abîmée et on en fait un paquet plat qui est ensuite discrètement dissimulé par un assistant dans un lieu secret. Semblable à un animal mort, une membrane hors d'usage est considérée comme impure (jutho), et la vue d'un tel «cadavre» provoque selon les Magar des impasses métaphysiques.

Comme dans d'autres ethnies himalayennes, on utilise pour la membrane la peau d'une chèvre sauvage, offerte au chamane ou au novice par les assistants. Avant de pouvoir être travaillée, la peau doit être séchée pendant quelques semaines dans une maison.

L'opération commence par le découpage de la peau selon la dimension du cadre. On la tend ensuite sur le cadre à l'aide de lanières de cuir trempées auparavant dans un bol en cuivre. Grâce à une alène et une pincette, les lanières sont passées dans les trous percés par le forgeron, de manière à former une ligne en zigzag sur le cerceau. Pendant l'opération, les participants consomment une couenne de buffle coupée en petits morceaux, censée les protéger contre le danger.

Plus tard, les assistants se mettent à épiler la peau tendue sur le cadre. A cette fin, elle est chauffée au feu jusqu'à ce que l'ouverture des pores permette d'arracher les poils. On ne laisse que quelques poils sur le bord pour rappeler l'origine animale de la membrane. Ensuite, la peau est rasée à l'aide d'un petit couteau. Le côté chair, toujours à l'intérieur du tambour, n'est pas du tout travaillé pendant ce rite.

En même temps, on confectionne des petits pains qui, disposés dans un trou, sont recouverts du tambour une fois que la membrane est fixée.'Ils sont destinés aux divinités des terres non cultivées (sepā et serõ). Puis le chamane officiant les donne à manger aux participants.

La membrane est ensuite peinte avec de la craie blanche dissoute dans un bol d'eau. On utilise les doigts trempés dans la craie. Les motifs peuvent varier: il existe des dessins astraux et cosmologiques, des dessins figuratifs comme des poissons, des tambours ou des organes sexuels en éjaculation, des dessins géométriques comme des triangles, des rhombes, des pentagones et des cercles, des improvisations abstraites faisant penser aux peintures de Pollock. Une fois accomplis ces gestes rituels dont chacun est accompagné par le chant du mythe correspondant, le chamane officiant se lève et commence une danse en s'accompagnant sur son propre tambour. Un assistant prend le nouveau tambour et l'imite. Ce n'est toujours pas le propriétaire du tambour ou un autre chamane qui effectue la consécration, mais un non-inité. Ensuite, le nouvel instrument est arrosé d'un mélange magique composé de grains, de cendres, de scories de cuivre et de silex, tandis que des mantras sont prononcés sur lui. 
La cérémonie est close par une dernière danse. Cette fois-ci le chamane officiant danse en cercle, soit dans le sens des aiguilles d'une montre, soit en sens inverse (symbolisant respectivement la vie et la mort). En même temps, un assistant fait rouler le nouveau tambour par terre en suivant exactement les mouvements du chamane. A ce moment, le tambour, futur véhicule du voyage chamanique, accomplit lui-même un trajet symbolique. Le même rituel est répété devant la maison du propriétaire du tambour. Mais c'est maintenant ce dernier qui fait rouler son tambour. Près du seuil, il le lâche: le tambour roule seul vers l'entrée, jusqu'aux mains de son épouse qui l'attrape et l'arrose de levure, comme le prescrit le mythe. Pour clore le rituel de consécration, un poussin est abattu dans la maison, et son sang versé par le chamane sur le cadre du tambour. La fabrication du tambour rẽ est maintenant terminée: il est consacré et prêt à l'usage.

$\mathrm{Si}$, dans le contexte sibérien, on assimile volontiers la consécration d'un nouveau tambour à une "animation", tel n'est pas le cas chez les Magar où la consécration vise essentiellement à apprivoiser la vitalité excessive, voire même la férocité du tambour neuf, à le pacifier et à maîtriser son potentiel extatique - comme il faut le faire aussi pour un jeune chamane encore inexpérimenté. C'est donc de l'«apaisement» et de la «domestication» partielle du tambour qu'il s'agit ici.

Aux yeux des Magar, le tambour $r e \tilde{n}$ 'est pas un objet inanimé mais vivant. Sa vie et sa biographie dépendent toutefois du chamane auquel il appartient. Sans lui, il ne peut pas «naître» en tant qu'instrument, c'est-à-dire passer d'une latte de chêne à un cadre de tambour. Comme on l'a vu, la découverte de l'arbre et la fabrication réussie du tambour sont elles-mêmes des étapes essentielles du processus initiatique. Pas de noviciat sans naissance et consécration préalables du tambour. Une fois que le novice a accédé à l'état d'élu, son tambour l'accompagnera durant toute sa vie professionnelle, que ce soit lors des séances de guérison, de l'initiation d'autres chamanes, de rencontres entre chamanes, de l'enterrement d'un confrère - ou de son propre enterrement.

A la mort d'un chamane, ses confrères l'enterrent en position assise sous un tumulus à l'extérieur du village. Un conifère, l'arbre de vie du chamane, abrite la tombe. Dans ses branches est suspendu l'équipement du défunt: sa couronne en plumes de faisan, des pendentifs et des cloches en fer, ainsi que son tambour dont on a déchiré la membrane; car au moment où le chamane expire, son compagnon, le tambour, doit se taire également. L'instrument reste suspendu pendant neuf jours, puis il est réclamé par la parenté du défunt qui le garde jusqu'à ce que son successeur réincarné le réclame. Ainsi le tambour se trouvet-il intégré, à travers la succession de ses propriétaires, dans le concept de la réincarnation: lui aussi peut renaître. Mais cela ne s'applique en pratique que dans les cas où le cadre peut encore servir. Indépendamment du fait de la transmission du tambour, chaque novice en reçoit un nouveau.

L'espérance de vie d'un tambour est de dix à quinze ans, celle d'une membrane de trois à cinq ans. Ensuite il faut les renouveler. Le procédé de réparation et de renouvellement d'un tambour est analogue à celui de la fabrication 
d'un nouveau tambour. L'usure d'un tambour, par exemple la déchirure de la membrane, ne provoque pas la mort de son propriétaire, comme le pensent notamment les Evenques (cf. Delaby 1976: 111). Cela indique notamment que le tambour n'est pas à concevoir, au sens strict, comme l'alter ego ou le sosie du chamane. Au cours de sa vie, un chamane peut posséder plusieurs tambours, ce qui n'enlève rien au fait que le tambour reste son compagnon le plus fidèle dans le cours de sa vie professionnelle.

Quelles sont les propriétés du tambour rẽ ? En tant qu'instrument magique, il contient des pouvoirs - généralement d'ordre surnaturel - qui sont absents d'autres instruments de musique et apparaissent selon ses fonctions rituelles.

\section{L'accompagnement de l'énoncé du mythe}

Du point de vue rituel, la principale fonction de l'instrument réside dans son rôle d'accompagnement des chants: le tambour rẽ rythme la narration des mythes d'origine et des récits mythiques versifiés exécutés lors des séances de guérison et d'initiation. Dans le cas des mythes cosmogoniques, il y a le plus souvent deux interprètes: un chanteur principal et maître expérimenté, accompagné par un disciple qui répète chaque vers et mémorise ainsi, au fil des années, l'immense répertoire de la tradition orale. Tous deux sont des chamanes initiés, et tous deux jouent de leur propre tambour. Dans le cas des mythes au contenu narratif complexe, le premier chanteur adopte souvent la pose d'un conteur faisant face au public, tandis qu'il revient au disciple de tomber en transe lors des passages appropriés.

Le rythme frappé sur le tambour est le même pour l'ensemble des mythes ainsi que pour la plupart des chants rituels auxiliaires. Il suit le mètre des vers et se présente ainsi ${ }^{7}$ :

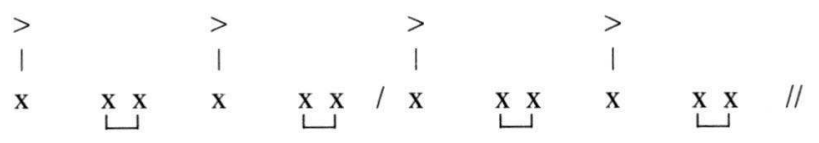

Cette combinaison entre le battement du tambour et la voix, entre le temps et le mètre constitue le modèle de base de quelque dix mille vers de la tradition orale des chamanes magar. Les chants primordiaux fournissent le prototype mythique des actes rituels accomplis dans le présent, que le tambour fixe au moyen de ses battements nomothétiques. 


\section{L'accompagnement de la danse}

Le tambour n'accompagne pas seulement le chant mais aussi la danse chamanique. La danse fait partie intégrante de tout événement rituel pour lequel le guérisseur revêt son attirail. Des représentations particulièrement spectaculaires ont lieu le jour principal de l'initiation, lorsque les guérisseurs doivent accomplir neuf rondes dansées entre la maison du novice et son arbre de vie érigé à l'extérieur du village, avant qu'il ne puisse naître rituellement sur l'arbre. A cette occasion, il se forme une longue file de danseurs (jusqu'à une trentaine) qui battent leur tambour à l'unisson et font cliqueter leur attirail. Les pas de danse se calquent ici sur le rythme des tambours.

Le rythme est aussi standardisé pour la danse que pour le chant, mais inversé:

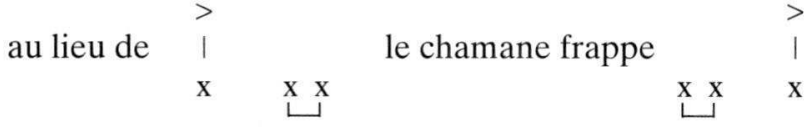

Ce rythme de base est aussi de rigueur pour les danses exécutées pendant les grandes séances de guérison. A ces occasions, le trajet de la danse mène de l'intérieur de la maison du patient sur le toit en terrasse d'une maison voisine, en passant par un escalier à neuf marches représentant l'échelle cosmique descendant dans le monde souterrain. Sur le toit et sous un panier renversé, est posée la «natte du monde» dont le guérisseur officiant fait neuf fois le tour pour symboliser un voyage aux confins du monde où il a dû suivre l'âme fuyante de son patient. Pendant la danse, le tambour entraîne donc le chamane dans un voyage rituel.

\section{Le voyage du chamane}

Le tambour que l'on fait «rouler» par terre pour signifier qu'il se déplace dans l'espace, et le tambour "chevauché» en tant que véhicule du voyage ont déjà été évoqués. S'y ajoutent les actes rituels accomplis de façon répétée au cours des séances de guérison nocturnes, qui ont pour thème un voyage symbolique du chamane. Ces voyages imaginaires portent divers noms, et leur durée est variable. L'un d'entre eux, destiné à la quête de l'âme, fait l'objet d'un chant intitulé «chant de la route» (dāwāta). D'après ce chant, le chamane poursuit l'âme fuyante de son patient jusqu'au col menant à l'autre monde, situé à la frontière orientale de l'aire tribale. Les nombreuses stations de ce périple sont marquées par le rythme du tambour. De temps à autre, le chamane s'arrête et regarde dans l'intérieur du tambour pour y voir, comme dans un miroir magique, les traces de l'âme et définir ainsi le trajet à suivre (fig. 3).

Pour le chamane, voyager avec son tambour signifie s'exposer aux dangers véhiculés par les forces néfastes: C'est pour se prémunir contre elles qu'il revêt 
son attirail. Mais auparavant, il invoque - toujours avec son tambour - l'aide des esprits auxiliaires, notamment l'esprit de son prédécesseur défunt et une série d'esprits animaux. L'«invocation d'un esprit ancestral» (pittr khulne), chant isolé du répertoire chamanique, est répété à plusieurs reprises au cours d'une séance. Par sa ligne mélodique et son rythme, il débute à la manière d'un mythe d'origine, même si du point de vue du contenu il y est question des prédécesseurs du chamane et de ses nombreux esprits auxiliaires aux formes d'animaux. Au moment où l'un de ceux-ci prend possession du chanteur, le battement du tambour devient irrégulier puis cesse complètement; le chanteur jette l'instrument, pousse des cris d'animaux et imite les mouvements caractéristiques de l'esprit qui s'est logé en lui. La descente des esprits auxiliaires requiert de toute évidence un grand effort du chamane - comme l'indiquent ses traits tendus; ceux-ci le propulsent dans l'extase, mais c'est seulement en les accommodant qu'il parvient à se défendre contre les attaques des forces néfastes.

\section{La communication avec les esprits}

Si le tambour permet d'appeler les bons esprits, il a aussi le pouvoir de repousser les mauvais. Cela s'accomplit à travers le bruit «cosmique» qu'il produit lorsqu'il est joué par le chamane qui danse. D'une part, ce bruit intimide les ennemis du guérisseur. D'autre part, au moyen du tambour et de la baguette, il est possible de clouer au sol les forces néfastes, en particulier les sorcières, et de les fixer ainsi définitivement. Selon la mythologie, la baguette symbolise ici alternativement le marteau qui frappe sur le tambour comme sur une enclume, et le clou qui attache les ennemis. Parfois même, elle est une arme offensive pour anéantir les sorcières, consœurs mythiques du chamane, en les précipitant du haut de la montagne.

Le tambour peut aussi être utilisé pour se défendre, en l'employant comme bouclier protégeant contre les flèches magiques invisibles lancées par les sorcières, les esprits de la chasse ou de la forêt, ou contre les ruses magiques de collègues malveillants. Par exemple, un récit local relate comment un rival du village voisin attira la foudre sur un chamane engagé dans un voyage rituel au bénéfice d'un patient. Du coin de l'œil, le guérisseur aperçut la foudre et parvint à la repousser grâce à son tambour dont le cadre se scinda en deux. C'est aussi une fonction protectrice qu'exerce le tambour lorsqu'il est utilisé pour couvrir des offrandes ou de la nourriture rituelle et les soustraire ainsi au regard envieux des esprits non conviés au festin.

Lors de certains voyages rituels, le chamane se rend dans le monde souterrain (tānje wānje) que la mythologie situe dans les plaines du sud. Arrivé là, il se transforme en esprit bénéfique du sanglier (galdevirr). Sous ce déguisement, il tombe dans un sommeil magique (sata). Mis à part quelques convulsions occasionnelles, il repose tranquillement par terre, la tête appuyée sur le tambour 


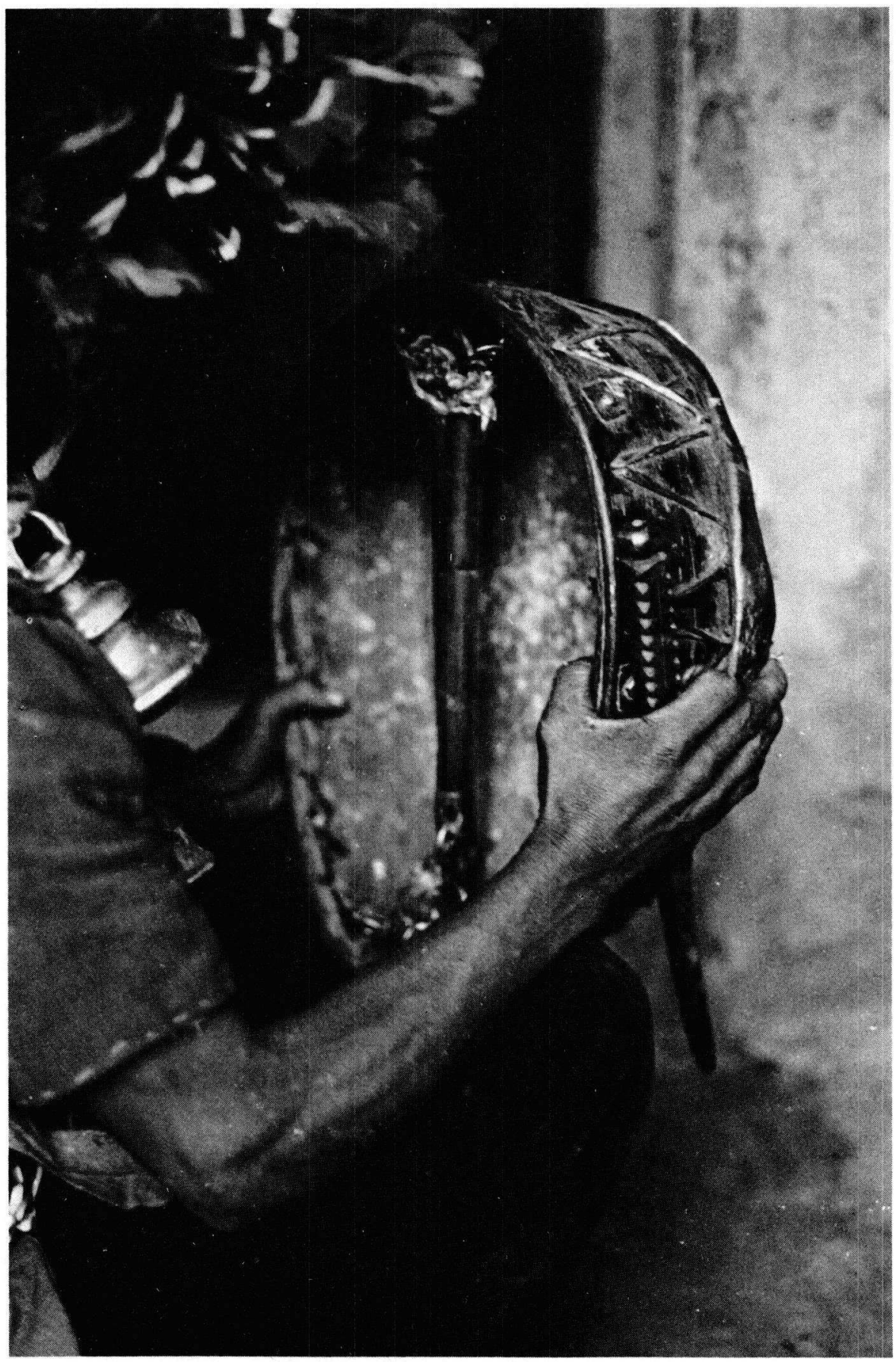

Fig. 3: Vision d'avenir à l'intérieur du tambour. Photo: M. Oppitz. 
dont le côté ouvert est en haut et le côté fermé en bas. De cette manière, le tambour est clos vis-à-vis du monde souterrain et réceptif face au monde terrestre. En appuyant sa tête sur son tambour, le guérisseur endormi est à même de «voir» l'âme fuyante de son patient. Dès qu'il la repère, il bondit, se met à quatre pattes devant l'arbre de vie attaché au poteau central de la maison de son patient et, toujours sous la forme de l'esprit du sanglier, il soulève avec un mouvement abrupt le patient placé sur son dos. Par ce geste appelé galsine ( «faire comme le sanglier») il ramène l'âme du patient du monde souterrain. Le second chamane présent dans ce rite frappe quelques coups secs sur son propre tambour qu'il tient au-dessus du patient, afin de clouer l'âme revenue dans le corps qui est le sien. Dans cette brève séquence, le tambour constitue à la fois le véhicule du voyage, de l'extase, de la clairvoyance et du rapatriement de l'âme.

\section{La divination}

Enfin, le tambour chamanique peut être employé pour pratiquer les deux modes de divination en usage localement. Dans le premier cas, le chamane en transe se trouve sous l'influence d'un esprit auxiliaire, et il énonce ses prédictions dans un langage spirituel appelé lämā kham, sorte de tibétain fictif compris seulement par les initiés, et qu'il faut traduire aux non-initiés. Cette forme de divination extatique (paĩsine) se pratique par exemple pour «reconnaître», en état de légère transe, les traces laissées par l'âme à l'intérieur du tambour, et pour «identifier» en état de sommeil magique son lieu de séjour sur la face ouverte du tambour.

Le second type de divination consiste en l'examen d'un objet, tel que: les entrailles d'un animal, des assiettes ou des tambours et des bûches enflammées jetés en l'air; c'est aussi l'interprétation de la voix des corbeaux et de la trajectoire de boulettes en tissu sur un tambour plat. Cette forme de divination est appelée pärche, terme apparenté au sanscrit parìkșā et signifiant «examen», «test», «interprétation» ou «interprétation de rêve». De cette catégorie relève une forme de divination impliquant le tambour, qui fait partie intégrante du rituel de chaque grande séance de guérison, à savoir le $r \bar{l}$ ranne ou $r \bar{l}$ khelne, «faire courir» ou «regarder la boule d'ordures». Le substantif $r \bar{l}$ signifie «saleté», «ordures», mais dans le présent contexte il s'applique à un morceau d'étoffe découpé dans les vêtements du patient, dont on forme une boule à l'aide de particules de la racine purifiante $t i$ et d'une feuille d'absinthe pour la placer ensuite sur la membrane d'un tambour tenu horizontalement (fig. 4). Le chamane fait vibrer la membrane en y portant des coups légers, et la boulette commence à sauter sur la surface du tambour jusqu'à ce qu'elle s'arrête à un certain point ou tombe. Chaque point du cerceau pouvant être soit de bon soit de mauvais augure, l'endroit où la boulette $\bar{r}$ tombe du tambour a sa propre valeur de prédiction. Le meilleur signe est que la boule reste sur la membrane. 


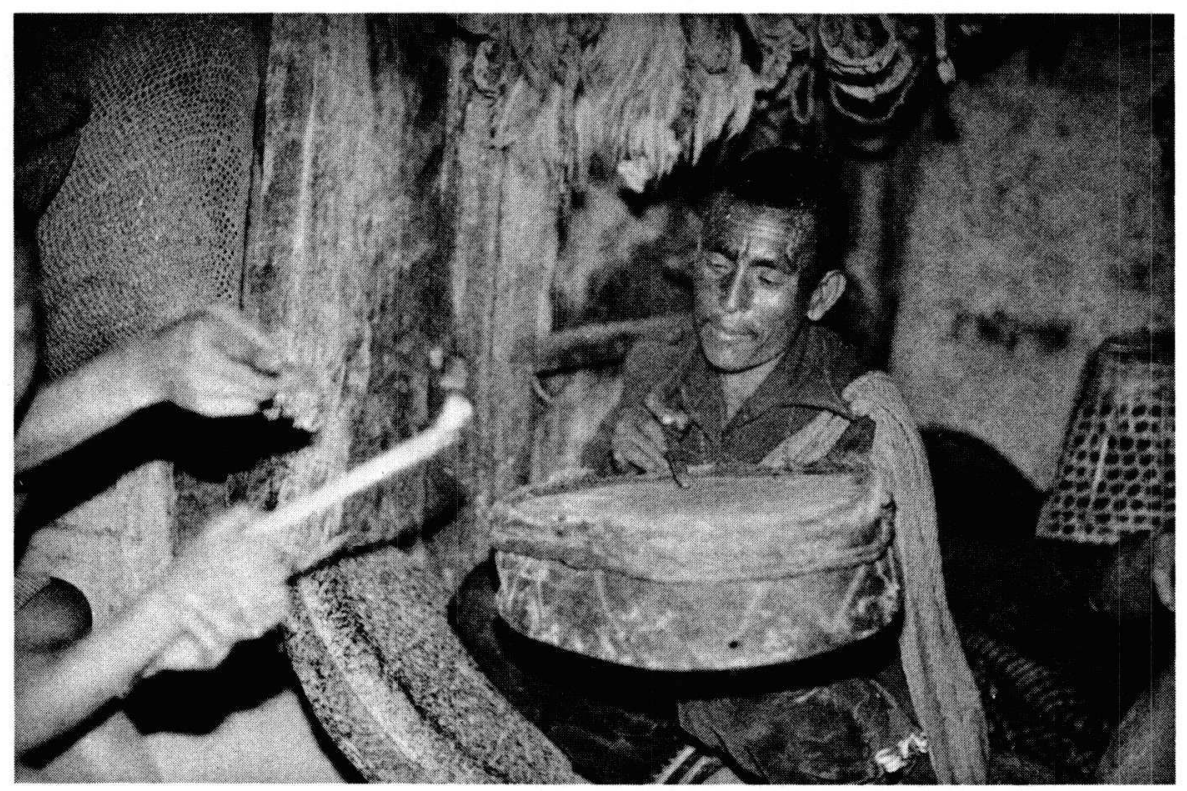

Fig. 4: Divination au moyen de boulettes d'ordures sur le tambour tenu horizontalement. Photo: M. Oppitz.

Le processus est répété trois fois, car toute combinaison comporte également une signification divinatoire. Si la boule tombe trois fois sur le même point, c'est bon signe, peu importe sa signification. Si elle tombe une fois sur un rivet en fer (toujours un point dangereux), une fois sur une lanière en cuir et une fois sur l'un des triangles formés par les lanières, c'est très mauvais signe. Après le troisième tour, le chamane s'écrie «höi» et comme pour terminer un chant, il frappe le cadre de son tambour de quelques brefs coups de baguette ${ }^{8}$.

Dans tous les cas examinés ci-dessus, le tambour rẽ apparaît comme un moyen de se transporter dans l'Au-delà, investi qu'il est de pouvoirs magiques. Ce qui n'est guère surprenant, car il est l'instrument des chamanes, ces médiateurs par excellence entre l'univers des hommes et l'univers non humain.

8 La divination avec le tambour et à l'aide d'objets mûs par vibration sur la membrane du tambour tenue horizontalement est aussi attestée dans d'autres régions de l'Himalaya. Chez les Tamang, on utilise des grains de riz (Mastromattei 1988: pl. 16), et au Tibet des grains de blé ou d'orge. Au Tibet, l'art de la divination avec le tambour a atteint un niveau élevé de raffinement (Nebesky-Wojkowitz 1952: 149-57). Mais la forme la plus célèbre de prédiction chamanique au moyen d'un tambour plat est attestée pour les anciens Sami. Dans ce cas, le tambour lapon était frappé avec un marteau double en corne ayant la forme d'un T, qui faisait bouger un «bras» en laiton, bois ou corne de renne sur la membrane (Manker 1938: 300-433). 


\section{Bibliographie}

BERGLIE Per Arne

1976 «Preliminary remarks on some Tibetan 'spiritual mediums' in Nepal». Kailash (Bhatahity) IV(1): 85-108.

DELABY Laurence

1976 Chamanes toungouses. Nanterre: Université de Paris X [Études mongoles et sibériennes, Cahier 7].

HASLUND-CHRISTENSEN Henning

1944 «Mongolske Troldmaend». Fra Nationalmuseets Arbejdsmark, Copenhague.

HEISSIG Walther

1944 «Schamanen und Geisterbeschwörer im Küriye-Banner». Folklore Studies 3: 39-72.

HELFFER Mireille

1983 «Observations concernant le tambour tibétain rnga et son usage». Selected Reports in Ethnomusicology $I V: 62-97$.

\section{HOFFMANN Helmut}

1950 Quellen zur Geschichte der tibetischen Bon-Religion. Mainz: Akademie der Wissenschaften und der Literatur. [Abhandlungen der Geistes- u. Sozialwissenschaftlichen Klasse Nr. 4]

LOT-FALCK Eveline

1961 «A propos d'un tambour de chaman toungouse». L'Homme I (2) : 23-50.

MANKER Ernst

1938/1958 Die lappische Zaubertrommel (2 vol.). Stockholm. [Acta Laponica I et II].

MASTROMATTEI Romano

1988 La terra reale. Rome: Ed. Valerio Levi.

NEBESKY-WOJKOWITZ René de

1952 «Tibetan drum divination, 'ngamo'». Ethnos 17: 149-57. 